\title{
Analysis of Overhead Transmission Line Parameters with Uncertainty
}

\author{
Yoseph Mekonnen Abebea ${ }^{\mathrm{a}}$, P. Mallikarjuna Rao ${ }^{\mathrm{b}}$, M. Gopichand Naik ${ }^{\mathrm{c}}$ \\ ${ }^{a}$ Dept. of Electrical Eng., College of Engineering (A), Andhra University, Visakhapatnam-530003, India \\ ${ }^{b}$ Dept. of Electrical Eng., College of Engineering (A), Andhra University, Visakhapatnam-530003, India \\ ${ }^{c}$ Dept. of Electrical Eng., College of Engineering (A), Andhra University, Visakhapatnam-530003, India
}

Received: 06 January 2017; Accepted: 13 February 2017; Published: 08 September 2017

\begin{abstract}
Overhead Transmissions Lines (OTL) are outdoor systems, which can easily be affected by weather fluctuation. In the worst-case scenario, temperature variation, horizontal wind and vertical ice-loading make the value of sag, tension and conductor (cable) length to vary between low to high extremes. The temperature and cable length variations have an effect on the resistance of the OTL. This variation leads to a variable voltage drop, which is mostly considered with load variation. In order to calculate resultant loading, sag, tension, cable length and resistances of an OTL, an uncertainty model based on Standard Affine Arithmetic (SAA) is proposed and the result is compared with the probabilistic Monte Carlo (MC) and Interval Arithmetic (IA) approaches. Based on the test results, the SAA based algorithm gives slightly conservative bound than the IA and MC approaches.
\end{abstract}

Index Terms: Interval Arithmetic, Monte Carlo, Overhead transmission line, Standard Affine Arithmetic, Uncertainty, Weather Change.

(C) 2017 Published by MECS Publisher. Selection and/or peer review under responsibility of the Research Association of Modern Education and Computer Science.

\section{Introduction}

Overhead transmission lines (OTLs) are a pathway for power transfer from the generation to substation and then to the customer side. The capability of an OTL depends on its strength against wind loading, ice loading and temperature variation [1]. The higher the wind pressure and the ice weight on the cable, the greater chance that the cable will break and transmission is disturbed. When the temperature increases, it has an effect not only on the cable breakage, but also on the current transmitted through the cable. The effect of the wind pressure and

* Corresponding author. Tel.: +919703758851

E-mail address: yosephgod@gmail.com, electricalprofessor@gmail.com,gopi_905@yahoo.co.in 
ice accumulation can result from increasing weight of the cable to a series of accidents like breakage of the line, falling of the tower or pole, creation of fire on forest and etc. The probability that the OTL can cause fire must be known in order to save life and property [2]. As a result, the worst-case effect of weather on the OTL must be dealt in detail before erecting the tower [3]. High wind blow and ice accumulation are much worse in cold regions. The accumulation of ice on the OTL happens suddenly when the temperature of the area is low. Sometimes, such accumulation of ice and wind pressure oscillate the cable from low to high amplitudes to the extent a flashover between cables can happen. The insulators and the towers may face huge impact due to the transient forces [4].

The variation of ice accumulation on the cable and asynchronous ice shading create a non-uniform ice loading which has a high impact on the tension of the OTL, to the extent of breaking the line or bending of the tower [5]. Local winds in the form of tornadoes or hudhud cyclones, like the one happened in 2014 in Visakhapatnam, India, may result full scale line and tower damage. Sometimes such cyclones and tornado effect on the OTL are beyond the damage of cables and towers. In 2014, in Visakhapatnam, India, initially when the cyclones start there was a flash over between the lines and then the wind pressure increased to the extent of making the overall OTL out of usage.

According to [6], the insulator length has less effect on the tower cross arm than the combined wind pressure, ice loading and the initial tension. The cross arms of the towers are initially designed to withstand both the wind pressure and ice loading, however with global weather changes the same area may face different loading than what has been predicted during the design stage.

OTLs play a great role for safe power delivery to the desired location. The initial design has to consider load capability of the line including environmental factors. Those factors and measuring devices value are not absolute rather they vary between low and high extreme seasonally and change from place to place [7]. A Weakly designed OTLs, which does not consider the worst-case scenario, are unable to deliver power to the substations reliably. This may lead to market loss or in the worst case it may results huge damage to the system.

Though weather change is the main source of variation of OTL parameters, the error coming from measurement sensors is also a non-ignorable input uncertainty. The measuring ability of the sensor is also affected by weather changes and the aging of devices which finally results an uncertain output [8].

The variation in OTL varies the voltage drop accordingly and the loss oscillates between high and low extreme resulting un reliable power delivery [9].

As a result of the aforementioned variation of ice loading, wind loading and temperature variation, the overall OTL parameters cannot be calculated with certainty. Though the uncertainty model is applied by some researchers to calculate power flow analysis and transient stability analysis, little attention has been given to the sources of the uncertainty and uncertain OTL modeling. In this paper, the uncertainty model for calculating OTL loading, sag, tension, cable length and worst case resistance value that use Standard Affine Arithmetic (SAA) has been proposed. The proposed algorithm is tested by using case studies and comparison is also made to validate the proposed model.

This paper is organized as follows: section two give an introduction about SAA with main mathematical principles; section three deals about the proposed mathematical model; section four is dedicated to result and discussions and finally section five gives the conclusion of the overall work.

\section{Standard Affine Arithmetic}

SAA is an extended or advanced version of Interval Arithmetic (IA) with the ability of tackling most of the drawbacks of IA. SAA like IA gives an enclosed output for the computed inputs. It takes into account all the uncertainties whether it is due to an input data error or round-off and truncation error happening during computation. Round-off and truncation errors are represented by unique symbolic variables when error happens $[10,11]$. Generally, for any function ' $\mathrm{g}$ ' and ' $\mathrm{y}$ ' which are bounded, real and having the same sources of uncertainty, the affine form is given by (1).

For any interval function $U$ bounded by $[u, \hat{u}]$ and an affine function $\hat{g}$ of $(1 b)$, the interval to affine form and 
the affine to interval form is given by (2). Equation (2a) shows interval to affine conversion and (2b) shows affine to interval form conversion.

$$
\left.\begin{array}{ll}
\hat{u}=u_{0}+\sum_{i=1}^{n} u_{i} \varepsilon_{i} & (a) \\
\hat{g}=g_{0}+\sum_{i=1}^{n} g_{i} \varepsilon_{i} & (b)
\end{array}\right\}
$$

In this paper, affine functions are represented by small letters and their converted interval values are represented by the same letter in capital form.

$$
\begin{aligned}
& \text { where } \\
& \hat{u}, \hat{g} \quad \text { Affine functions } \\
& u_{0}, g_{0} \quad \text { Central values } \\
& u_{i}, g_{i} \quad \text { Partial deviations } \\
& \varepsilon_{i} \quad \text { Symbolic variables: }-1 \leq \varepsilon_{\mathrm{i}} \leq 1 \\
& \left.\begin{array}{ll}
\hat{u}=\frac{\bar{u}+\underline{u}}{2}+\frac{\bar{u}-\underline{u}}{2} \varepsilon_{i} & (a) \\
G=g_{0}+\sum_{i=1}^{n} g_{i}[-1,1] & \text { (b) }
\end{array}\right\}
\end{aligned}
$$

Ordinary operations in affine arithmetic are divided into affine and non-affine operations. Those operations which do not result in round-off and truncation errors are categorized under affine operations and those which can be rounded-off and truncated are categorized under non-affine operations [10-13]. The basic affine and non-affine operations are formulated based on the affine functions in (1) and the main affine operations are given by (3). Non-affine operations, like multiplication and division are approximated rather than directly analyzed. Multiplication, which is the most known non-affine operation is approximated by (4). Generally, any non-affine operations on a single function can be approximated by Chebyshev formula given by (5). The coefficients of (5) can be found from [10,11, 14].

$$
\left.\begin{array}{c}
a \hat{u}=a u_{0}+\sum_{i=1}^{n} a u_{i} \varepsilon_{i} \\
a \pm \hat{u}=\left(a \pm u_{0}\right)+\sum_{i=1}^{n} u_{i} \varepsilon_{i} \\
\hat{u} \pm \hat{g}=\left(u_{0} \pm g_{0}\right)+\sum_{i=1}^{n}\left(u_{i} \pm g_{i}\right) \varepsilon_{i} \\
\hat{u} \hat{g}=\left(u_{0} g_{0}\right)+\sum_{n=1}^{n}\left(u_{0} g_{i}+u_{i} g_{0}\right) \varepsilon_{i} \\
+\left(\sum_{i=1}^{n}\left|u_{i}\right| \sum_{i=1}^{n}\left|g_{i}\right|\right) \varepsilon_{n+1}
\end{array}\right\}
$$

In (3) $\alpha$ is a real number and in (5) the unique symbolic variable, $\varepsilon_{n+1}$ is distinct from symbolic variable $\varepsilon_{\mathrm{i}}$. 
Any differentiable and continuous function within its bound can be approximated by (5) for any non-affine operation on it $[11,14]$.

$$
\hat{z}=\alpha \hat{u}+\xi+\delta \varepsilon_{n}
$$

\section{Modeling OTL using SAA}

The presence of uncertainty in OTL leads to the formulation of uncertainty based OTL analysis. The starting point in the analysis of any system using SAA is based on the basic equations that describe the system. In this section, the basic OTL analysis is dealt first and then the SAA based OTL analysis is formulated based on the basic governing OTL equations.

\subsection{Basics of OTL}

A short span OTL has the shape of a parabola as shown in Fig 1. All the OTL parameters, namely, total loading, cable sag, tension and cable length of a parabolic shaped OTL are developed based on Fig 1. In the figure, it is assumed that one half of the parabola is the mirror image of the other half. In other words, the two halves are symmetric to each other. All the basic equations for any short span OTL with parabolic shape are derived from this assumption [15].

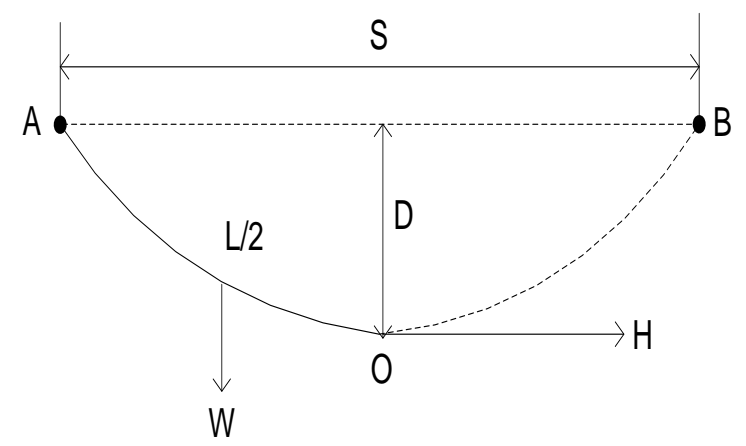

Fig.1. Short Span OTL (where A and B are the end of the span, O is the centre, S is the span length, L is the conductor length, $\mathrm{H}$ is the horizontal tension, $\mathrm{D}$ is the sag and $\mathrm{W}$ is the weight of the Bare Conductor)

The finding of the total loading of the cable, which is the result of, ice accumulation, wind pressure and bare cable weight is based on vector summation. The weight of bare cable and ice loading with an ice density of 57 $\mathrm{lb} / \mathrm{ft}^{3}$ are vertically downward from the center of the total mass. On the other hand, the wind pressure creates a horizontal force on the cable and results a horizontal loading. The resultant weight is then found by using the principle of vector addition [16]. Equation (6) contains the ice, wind and resultant loading per unit length of a cable respectively.

$$
\left.\begin{array}{ll}
W_{\text {ice }}=1.244 t\left(D_{c}+t\right) & (a) \\
W_{\text {wind }}=P_{w}\left(\frac{D_{c}+2 t}{12}\right) & (b) \\
W_{R}=\sqrt{\left(W_{b}+W_{i}\right)^{2}+W_{w}^{2}}+k & (c)
\end{array}\right\}
$$


where

$\begin{array}{ll}\mathrm{W}_{\text {ice }} & \text { Ice loading }(\mathrm{lb} / \mathrm{ft}) \\ \mathrm{W}_{\text {wind }} & \text { Wind loading }(\mathrm{lb} / \mathrm{ft}) \\ \mathrm{W}_{\mathrm{R}} & \text { Resultant weight }(\mathrm{lb} / \mathrm{ft}) \\ \mathrm{W}_{\mathrm{b}} & \text { Bare cable weight }(\mathrm{lb} / \mathrm{ft}) \\ \mathrm{t} & \text { Ice thickens }(\mathrm{in}) \\ \mathrm{D}_{\mathrm{c}} & \text { Diameter of the bare cable }(\mathrm{ft}) \\ \mathrm{P}_{\mathrm{w}} & \text { Wind pressure }\left(\mathrm{lb} / \mathrm{ft}^{2}\right)\end{array}$

The wind pressure in (6b) can be found from (7), where $V_{\text {wind }}$ is the blowing wind speed in (mph).

$$
P_{w}=0.0025\left(V_{\text {wind }}^{2}\right)
$$

Temperature variation changes the sag from $\mathrm{D}_{0}$ to $\mathrm{D}$ according to $(8)$ and the cable length from $\mathrm{L}_{0}$ to $\mathrm{L}$ according to (9). The combined change of the cable length due to thermal expansion and elastic elongation is given by (10).

$$
\begin{aligned}
& D_{0}=\frac{W_{R} S^{2}}{8 H_{0}} \quad(a) \\
& D=\frac{W_{R} S^{2}}{8 H}
\end{aligned}
$$

where

$$
\begin{array}{ll}
\mathrm{D}_{0} & \text { Initial sag }(\mathrm{ft}) \\
\mathrm{D} & \text { Final sag }(\mathrm{ft}) \\
\mathrm{H}_{0} & \text { Initial tension (lbs) } \\
\mathrm{H} & \text { Final tension (lbs) } \\
L_{0}=S\left(1+\frac{8 D_{0}^{2}}{3 S^{2}}\right) \quad(a) \\
\left.\begin{array}{ll}
L=S\left(1+\frac{8 D^{2}}{3 S^{2}}\right) & (b)
\end{array}\right\}
\end{array}
$$

where

$\mathrm{L}_{0} \quad$ Initial cable length

L $\quad$ Final Cable length

$$
L=L_{0}\left(1+\alpha_{T}\left(T-T_{0}\right)\right)\left(1+\frac{H-H_{0}}{E A}+e_{c}\right)
$$

where

$\alpha_{\mathrm{T}} \quad$ Thermal expansion coefficient,

$\mathrm{T} \quad$ Final temperature $\left({ }^{0} \mathrm{C}\right)$,

$\mathrm{T}_{0} \quad$ Initial temperature $\left({ }^{0} \mathrm{C}\right)$,

$\mathrm{e}_{\mathrm{c}} \quad$ Creep constant

E Elastic modulus (MPsi)

A Cable area $\left(i^{2}\right)$ 
Substituting (9) into (10) results (11) and it can be solved by a root finding mechanism of a cubic polynomial function. The coefficients $\Gamma_{1}, \Gamma_{2}, \Gamma_{3}$ and $\Gamma_{4}$ are given by (12). Using the loading result of (6c), the cubic function in (11) is used to find the final tension values. Among the three roots of (11), only one root has a real and positive value and it is considered as the tension of the OTL. For short span OTL with a shape of parabola shown in Fig 1, the tension ' $T$ ' is the same as the horizontal tension ' $H$ ' $[16,17]$.

The fixed system model, (6-12) is only applicable when there is no consideration of uncertainty. If uncertainty is considered in calculating OTL sag, cable length, Tension, weight or impedance of the line, the fixed input parameter model must be extended to an interval input based analysis [15-17].

$$
\left.\begin{array}{c}
\Gamma_{1} H^{3}+\Gamma_{2} H^{2}+\Gamma_{3} H-\Gamma_{4}=0 \\
\Gamma_{1}=\left(1+\frac{W_{R}^{2} S^{2}}{24 H_{0}^{2}}\right)\left(1+\alpha_{T}\left(T-T_{0}\right)\right)\left(\frac{1}{E A}\right) \\
\Gamma_{2}=\left(1+\frac{W_{R}^{2} S^{2}}{24 H_{0}^{2}}\right)\left(1+\alpha_{T}\left(T-T_{0}\right)\right)\left(1-\frac{H_{0}}{E A}+e_{c}\right)-1 \\
\Gamma_{3}=0 \\
\Gamma_{4}=\frac{W_{R}^{2} S^{2}}{24 H_{0}^{2}}
\end{array}\right\}
$$

\subsection{SAA based Analysis of OTL}

SAA based analysis of OTL is modeled considering the presence of uncertainty in ice thickness and wind pressure which is caused by temperature variation. For a bounded ice thickness and wind pressure, by upper and lower bound, their SAA form is given by (13a) and (13b) respectively.

$$
\left.\begin{array}{ll}
\hat{t}_{i}=t_{i, 0}+\sum_{n=1}^{k} t_{i, n} \varepsilon_{t, n} & (a) \\
\hat{P}_{w}=P_{w, 0}+\sum_{n=1}^{k} P_{w, n} \varepsilon_{p, n} & (b)
\end{array}\right\}
$$

where $\hat{t}_{\mathrm{i}}$ and $\hat{\mathrm{P}}_{\mathrm{i}}$ are affine function, $\mathrm{t}_{\mathrm{i}, 0}$ and $\mathrm{P}_{\mathrm{w}, 0}$ are central terms, $\mathrm{t}_{\mathrm{i}, \mathrm{n}}$ and $\mathrm{P}_{\mathrm{w}, \mathrm{n}}$ are partial deviations. The noise symbol $\varepsilon_{t, n}$ and $\varepsilon_{p, n}$ represents the pertaining uncertainties of the ice thickness and the wind pressure respectively.

Substituting (13a) into (6a) and (6b), (13b) into (6b) then applying affine and non-affine operations found in (1-5) and finally substituting those results into (6c) brings the corresponding SAA based loading as given by (14).

$$
\left.\begin{array}{ll}
\hat{W}_{i}=w_{i, 0}+\sum_{n=1}^{k} w_{i t, n} \varepsilon_{t, n}+\sum_{n=1} w_{i e, n} \varepsilon_{i, n} & (a) \\
\hat{W}_{w}=w_{w, 0}+\sum_{n=1}^{k} w_{w p, n} \varepsilon_{p, n}+\sum_{n=1}^{k} w_{w t, n} \varepsilon_{t, n}+\sum_{n=1} w_{w e, n} \varepsilon_{w, n} & (b) \\
\hat{W}_{R}=w_{R, 0}+\sum_{n=1}^{k} w_{R p, n} \varepsilon_{p, n}+\sum_{n=1}^{k} w_{R t, n} \varepsilon_{t, n}+\sum_{n=1} w_{\mathrm{Re}, n} \varepsilon_{R, n} & (c)
\end{array}\right\}
$$


The size of the approximation error depends on the number of non-affine operations. Each non-affine operation results at least one new symbolic variable.

Where, $\hat{\mathrm{W}}_{\mathrm{i}}, \hat{\mathrm{W}}_{\mathrm{w}}, \hat{\mathrm{W}}_{\mathrm{R}}$ and $\mathrm{W}_{\mathrm{i}, 0} \mathrm{WW}_{\mathrm{W}, 0} \mathrm{~W}_{\mathrm{R}, 0}$ are affine forms and central values of the ice, wind and resultant loading of (14a-14c) respectively. Wit, $n,\left(\mathrm{~W}_{\mathrm{wp}, \mathrm{n}}\right.$ and $\left.\mathrm{W}_{\mathrm{wt}, \mathrm{n}}\right),\left(\mathrm{W}_{\mathrm{RP}, \mathrm{n}}\right.$ and $\left.\mathrm{W}_{\mathrm{Rt}, \mathrm{n}}\right)$ are the partial deviations of the ice, wind and resultant loading of (14a-14c) respectively. Wie,n , Wwe,n, $\mathrm{W}_{\mathrm{Re}, \mathrm{n}}$, and $\varepsilon_{\mathrm{i}, \mathrm{n}}, \varepsilon_{\mathrm{w}, \mathrm{n}} \varepsilon_{\mathrm{R}, \mathrm{n}}$ are partial deviation of the new symbolic variables and the new noise variables of the ice, wind and resultant loading of $(14 \mathrm{a}-14 \mathrm{c})$ respectively. The symbolic variable $\varepsilon_{\mathrm{R}, \mathrm{n}}$ contains $\varepsilon_{\mathrm{i}, \mathrm{n}}, \varepsilon_{\mathrm{w}, \mathrm{n}}$ and new approximation errors.

Once the resultant loading is known, the next step is evaluation of (11) at each boundary value of the resultant weight at the respective temperatures. Evaluating the cubic root of (11) results a boundary value of horizontal tension and after conversion by using (2a), it becomes as shown in (15).

$$
\hat{H}_{c}=h_{c, 0}+\sum_{n=1}^{k} h_{c, n} \varepsilon_{h, n}
$$

where $\hat{H}_{c}$ is the affine form, $h_{c, o}$ is the central value, $h_{c, n}$ is the partial deviation and $\varepsilon_{\mathrm{h}, \mathrm{n}}$ is the symbolic variable of the tension.

For short span OTL with the shape of parabola, the horizontal tension is the same as tension T. As a result, once the horizontal tension is known, the sag of the line can be found by inserting the resultant loading of (14) and the tension of (15) into (8b) and applying affine and non-affine operation of (1-5). The final sag of the line is then given by (16).

$$
\hat{D}_{c}=d_{c, 0}+\sum_{n=1}^{k} d_{c p, n} \varepsilon_{p, n}+\sum_{n=1}^{k} d_{c t, n} \varepsilon_{t, n}+\sum_{n=1}^{k} d_{c h, n} \varepsilon_{h, n}+\sum_{n=1} d_{c e, n} \varepsilon_{s, n}
$$

where $\hat{D}_{c}$ represents the affine form, $d_{c, 0}$ represents central value, $d_{c p, n}, d_{c t, n}, d_{c h, n}$ and $d_{c e, n}$ represents the partial deviations due to wind pressure, ice thickness, tension and non-affine operations respectively. The symbolic variable due to non-affine operation, $\varepsilon_{\mathrm{s}, \mathrm{n}}$, contains the new and all approximation errors previously found. Once the sag is known the cable length of the OTL can be found directly by inserting (16) into (9b) with the constants and applying the affine and non-affine operation in (1-5) results (17).

$$
\hat{L}_{c}=l_{c, 0}+\sum_{n=1}^{k} l_{c p, n} \varepsilon_{p, n}+\sum_{n=1}^{k} l_{c t, n} \varepsilon_{t, n}+\sum_{n=1}^{k} l_{c h, n} \varepsilon_{h, n}+\sum_{n=1} l_{c e, n} \varepsilon_{l, n}
$$

where $\hat{\mathrm{L}}_{\mathrm{c}}$ represents the affine form of the cable length, $1_{\mathrm{c}, 0}$ represents central value, $1_{\mathrm{cp}, \mathrm{n}}, 1_{\mathrm{ct}, \mathrm{n}}, 1_{\mathrm{ch}, \mathrm{n}}$ and $1_{\mathrm{ce}, \mathrm{n}}$ represents the partial deviations due to wind pressure, ice thickness tension and non-affine operations of the OTL cable length respectively. Similar to (16) symbolic variable, $\varepsilon_{1, \mathrm{n}}$ contains the new and all approximation errors previously calculated in finding the length of the cable.

\section{Results and Discussions}

In order to validate the proposed SAA based OTL parameter calculation, in the presence of input uncertainty, a test case containing twenty-four different temperature variations are used. The temperature is selected between two extremes below the freezing point of water and the corresponding wind pressure and ice thickness is tabulated accordingly as shown in table I. A flat $20 \%$ of uncertainty diameter is considered on the temperature, wind pressure and ice thickness. A drake conductor is used to verify the proposed algorithm.

The main constants, which are used in finding all the variables for Drake conductors, are as follows: 
$\mathrm{A}=0.7264 \mathrm{in}^{2}, \mathrm{~S}=700 \mathrm{ft}, \mathrm{W}_{\mathrm{b}}=1.094 \mathrm{lb} / \mathrm{ft}, \mathrm{E}=11.2 \mathrm{Mpsi}, \alpha_{\mathrm{T}}=10.6^{*} 10-6 / 0 \mathrm{~F}, \mathrm{H}_{0}=7875 \mathrm{lb}, \mathrm{e}_{\mathrm{c}}=0, \mathrm{k}=0$. The DC and AC resistance of the ASCR Drake conductor at $68{ }^{\circ} \mathrm{F}$ and $167{ }^{\circ} \mathrm{F}$, are $0.0214 \mathrm{~m} \Omega / \mathrm{ft}$ and $0.0263 \mathrm{~m} \Omega / \mathrm{ft}$ respectively. The temperature coefficient of resistance is $0.002383 /{ }^{\circ} \mathrm{F}$. The initial tension is chosen according to the NESC recommendations limit with the worst-case condition value which is found in [15]. The resistance value from the temperature change, can be calculated using the formula: $\mathrm{R}=\mathrm{R}_{0}\left(1+\alpha_{\mathrm{T}} \Delta \mathrm{T}\right)$ and tabulated along with input variables in table $1 . \mathrm{R}_{0}$ is the $\mathrm{DC}$ and $\mathrm{AC}$ resistance value given at $68^{\circ} \mathrm{F}$ and $167^{\circ} \mathrm{F}$ respectively.

It is clear that nothing is certain in the temperature varying environment for OTL and thus adapting a model which considers such uncertainty is mandatory. The question to be answered is whether the proposed method is effective in considering uncertainty than tradition mechanism or not. In order to answer such a question, a Monte Carlo (MC) and Interval arithmetic (IA) based algorithms are used for comparison purpose. All the three mechanisms are implemented using MATLAB. The MC approach is simulated for 10,000 trial values in order to get non-varying results. Table 2 shows the resultant weight of the three algorithms. The resultant weight is the combination of the bare cable weight, the ice and wind loading. As mentioned earlier, the affine form of the total weight can be found from (14c).

Table 1. Initial inputs and Resistance Changes

\begin{tabular}{|c|c|c|c|c|c|}
\hline \multirow[b]{2}{*}{$\begin{array}{l}\text { Test } \\
\text { Cases }\end{array}$} & \multicolumn{3}{|c|}{ Uncertain Parameters } & \multirow{2}{*}{$\begin{array}{l}\text { DC Resistance Change } \\
\text { Resistance }(\mathrm{m} \Omega / \mathrm{ft})\end{array}$} & \multirow{2}{*}{$\begin{array}{l}\text { AC Resistance Change } \\
\text { Resistance }(\mathrm{m} \Omega / \mathrm{ft})\end{array}$} \\
\hline & $\begin{array}{l}\text { Temperature } \\
\left({ }^{\circ} \mathbf{F}\right)\end{array}$ & $\begin{array}{l}\text { Ice Thickness } \\
\text { (in) }\end{array}$ & $\begin{array}{l}\text { Wind } \\
\text { Pressure(lb) }\end{array}$ & & \\
\hline 1 & -31 & 1.20 & 0.2 & {$[0.026607,0.026291]$} & {$[0.038905,0.038517]$} \\
\hline 2 & -29 & 1.15 & 0.4 & {$[0.026495,0.026199]$} & {$[0.038767,0.038404]$} \\
\hline 3 & -27 & 1.10 & 0.6 & {$[0.026383,0.026108]$} & {$[0.038629,0.038291]$} \\
\hline 4 & -25 & 1.05 & 0.8 & {$[0.026271,0.026016]$} & {$[0.038492,0.038178]$} \\
\hline 5 & -23 & 1.00 & 1.2 & {$[0.026159,0.025924]$} & {$[0.038354,0.038065]$} \\
\hline 6 & -21 & 0.95 & 1.4 & {$[0.026046,0.025832]$} & {$[0.038216,0.037953]$} \\
\hline 7 & -19 & 0.90 & 1.6 & {$[0.025934,0.025740]$} & {$[0.038078,0.037840]$} \\
\hline 8 & -17 & 0.85 & 1.8 & {$[0.025822,0.025649]$} & {$[0.037940,0.037727]$} \\
\hline 9 & -15 & 0.80 & 2.0 & {$[0.025710,0.025557]$} & {$[0.037802,0.037614]$} \\
\hline 10 & -13 & 0.75 & 2.2 & {$[0.025598,0.025465]$} & {$[0.037664,0.037501]$} \\
\hline 11 & -11 & 0.70 & 2.4 & {$[0.025485,0.025373]$} & {$[0.037526,0.037388]$} \\
\hline 12 & -9 & 0.65 & 2.6 & {$[0.025373,0.025281]$} & {$[0.037388,0.037276]$} \\
\hline 13 & -7 & 0.60 & 2.8 & {$[0.025261,0.025190]$} & {$[0.037250,0.037163]$} \\
\hline 14 & -5 & 0.55 & 3.0 & {$[0.025149,0.025098]$} & {$[0.037113,0.037050]$} \\
\hline 15 & -3 & 0.50 & 3.2 & {$[0.025037,0.025006]$} & {$[0.036975,0.036937]$} \\
\hline 16 & -1 & 0.45 & 3.4 & {$[0.024924,0.024914]$} & {$[0.036837,0.036824]$} \\
\hline 17 & 1 & 0.40 & 3.6 & {$[0.024822,0.024812]$} & {$[0.036711,0.036699]$} \\
\hline 18 & 3 & 0.35 & 3.8 & {$[0.024731,0.024700]$} & {$[0.036599,0.036561]$} \\
\hline 19 & 5 & 0.30 & 4.0 & {$[0.024639,0.024588]$} & {$[0.036486,0.036423]$} \\
\hline 20 & 7 & 0.25 & 4.2 & {$[0.024547,0.024476]$} & {$[0.036373,0.036285]$} \\
\hline 21 & 9 & 0.20 & 4.4 & {$[0.024455,0.024363]$} & {$[0.036260,0.036147]$} \\
\hline 22 & 11 & 0.15 & 4.6 & {$[0.024363,0.024251]$} & {$[0.036147,0.036009]$} \\
\hline 23 & 13 & 0.10 & 4.8 & {$[0.024271,0.024139]$} & {$[0.036034,0.035871]$} \\
\hline 24 & 15 & 0.005 & 5.0 & {$[0.024180,0.024027]$} & {$[0.035922,0.035734]$} \\
\hline
\end{tabular}

As shown in Table 2, the SAA based result is slightly inclusive of both MC and IA results. Since the aim of uncertainty based analysis is finding the worst-case results, as opposed to the punctual method in (6-12), the slight inclusiveness can be considered as one of the benefits of SAA.

Table 3-5 shows the OTL tension, sag and cable length respectively. The tension of the OTL is affected by the total weight of the cable and the temperature changes. Depending on their values, one is dominant over the other. 
Table 2. OTL Weight

\begin{tabular}{lrrr}
\hline \multirow{2}{*}{ Test Cases } & Weight per Span $(\mathrm{lb})$ & & \\
\cline { 2 - 4 } MC & IA & MC \\
\hline 1 & {$[3.8377,4.8412]$} & {$[3.8375,4.8413]$} & {$[3.7659,4.8749]$} \\
2 & {$[3.6665,4.5999]$} & {$[3.6661,4.6000]$} & {$[3.6008,4.6304]$} \\
3 & {$[3.5007,4.3669]$} & {$[3.5003,4.3670]$} & {$[3.2863,4.1671]$} \\
4 & {$[3.3403,4.1419]$} & {$[3.3400,4.1424]$} & {$[3.1396,3.9529]$} \\
5 & {$[3.1894,3.9306]$} & {$[3.1882,3.9308]$} & {$[2.9957,3.7432]$} \\
6 & {$[3.0396,3.7222]$} & {$[3.0394,3.7235]$} & {$[2.8569,3.5417]$} \\
7 & {$[2.8976,3.5235]$} & {$[2.8960,3.5243]$} & {$[2.7232,3.3485]$} \\
8 & {$[2.7583,3.3314]$} & {$[2.7579,3.3332]$} & {$[2.5944,3.1635]$} \\
9 & {$[2.6261,3.1483]$} & {$[2.6252,3.1500]$} & {$[2.4706,2.9865]$} \\
10 & {$[2.4980,2.9743]$} & {$[2.4977,2.9748]$} & {$[2.3517,2.8174]$} \\
11 & {$[2.3764,2.8069]$} & {$[2.3754,2.8073]$} & {$[2.2376,2.6561]$} \\
12 & {$[2.2588,2.6461]$} & {$[2.2583,2.6475]$} & {$[2.1282,2.5026]$} \\
13 & {$[2.1468,2.4940]$} & {$[2.1461,2.4953]$} & {$[2.0236,2.3565]$} \\
14 & {$[2.0396,2.3493]$} & {$[2.0389,2.3504]$} & {$[1.9235,2.2177]$} \\
15 & {$[1.9380,2.2115]$} & {$[1.9366,2.2126]$} & {$[1.8280,2.0860]$} \\
16 & {$[1.8392,2.0810]$} & {$[1.8390,2.0819]$} & {$[1.7368,1.9613]$} \\
17 & {$[1.7465,1.9579]$} & {$[1.7460,1.9580]$} & {$[1.6500,1.8433]$} \\
18 & {$[1.6587,1.8390]$} & {$[1.6576,1.8407]$} & {$[1.5675,1.7317]$} \\
19 & {$[1.5742,1.7291]$} & {$[1.5736,1.7297]$} & {$[1.4146,1.5271]$} \\
20 & {$[1.4948,1.6243]$} & {$[1.4939,1.6249]$} & {$[1.3441,1.4336]$} \\
21 & {$[1.4194,1.5256]$} & {$[1.4184,1.5260]$} & {$[1.2776,1.3457]$} \\
22 & {$[1.3475,1.4324]$} & {$[1.3470,1.4329]$} & {$[1.1574,1.1876]$} \\
23 & {$[1.2798,1.3447]$} & {$[1.2796,1.3452]$} & \\
24 & {$[1.1582,1.1875]$} & {$[1.1581,1.1875]$} & \\
\hline
\end{tabular}

Table 3. OTL Tension

\begin{tabular}{|c|c|c|c|}
\hline \multirow[t]{2}{*}{ Test Cases } & \multicolumn{3}{|l|}{ Tension (lb) } \\
\hline & $\mathrm{MC}$ & IA & AA \\
\hline 1 & {$[8723.7,8365.5]$} & {$[8723.8,8365.4]$} & {$[8757.1,8358.6]$} \\
\hline 2 & {$[8781.6,8406.7]$} & {$[8781.8,8406.7]$} & {$[8815.6,8399.6]$} \\
\hline 3 & {$[8843.5,8452.0]$} & {$[8843.7,8451.9]$} & {$[8877.8,8444.7]$} \\
\hline 4 & {$[8909.4,8501.8]$} & {$[8909.6,8501.6]$} & {$[8943.9,8494.2]$} \\
\hline 5 & {$[8976.6,8554.5]$} & {$[8977.4,8554.4]$} & {$[9011.8,8546.8]$} \\
\hline 6 & {$[9050.4,8613.9]$} & {$[9050.6,8613.4]$} & {$[9084.7,8605.7]$} \\
\hline 7 & {$[9125.9,8678.2]$} & {$[9127.3,8677.8]$} & {$[9161.0,8670.0]$} \\
\hline 8 & {$[9207.0,8748.8]$} & {$[9207.4,8747.9]$} & {$[9240.3,8740.0]$} \\
\hline 9 & {$[9289.2,8824.8]$} & {$[9290.1,8823.8]$} & {$[9322.1,8815.9]$} \\
\hline 10 & {$[9374.5,8905.9]$} & {$[9374.8,8905.6]$} & {$[9405.6,8897.8]$} \\
\hline 11 & {$[9459.5,8993.6]$} & {$[9460.8,8993.3]$} & {$[9489.9,8985.7]$} \\
\hline 12 & {$[9546.1,9087.8]$} & {$[9546.7,9086.6]$} & {$[9574.1,9079.2]$} \\
\hline 13 & {$[9630.6,9186.2]$} & {$[9631.5,9184.9]$} & {$[9656.8,9178.0]$} \\
\hline 14 & {$[9712.7,9288.6]$} & {$[9713.7,9287.5]$} & {$[9736.8,9281.0]$} \\
\hline 15 & {$[9789.6,9394.6]$} & {$[9791.8,9393.2]$} & {$[9812.5,9387.2]$} \\
\hline 16 & {$[9863.9,9501.7]$} & {$[9864.2,9500.5]$} & {$[9882.4,9495.1]$} \\
\hline 17 & {$[9937.2,9600.6]$} & {$[9937.9,9600.4]$} & {$[9953.7,9595.8]$} \\
\hline 18 & {$[10011,9691.6]$} & {$[10013,9689.1]$} & {$[10026,9685.2]$} \\
\hline 19 & {$[10079,9771.7]$} & {$[10080,9770.8]$} & {$[10091,9767.6]$} \\
\hline 20 & {$[10136,9844.3]$} & {$[10138,9843.2]$} & {$[10147,9840.7]$} \\
\hline 21 & {$[10184,9905.0]$} & {$[10186,9904.3]$} & {$[10193,9902.5]$} \\
\hline 22 & {$[10221,9953.0]$} & {$[10222,9952.3]$} & {$[10228,9951.1]$} \\
\hline 23 & {$[10247,9986.5]$} & {$[10247,9985.7]$} & {$[10251,9985.0]$} \\
\hline 24 & {$[10367,10133]$} & {$[10367,10133]$} & {$[10369,10132]$} \\
\hline
\end{tabular}


In table 3, the higher bound corresponds to the first row for each algorithm and it is due to the lower temperature and the upper weight. Similarly, the lower bound corresponds to the second row for the three algorithms and it is due to the upper bound temperature and lower bound weight. Similarly, as shown in table 3 the SAA result is slightly conservative than both MC and IA approaches.

The sag and conductor length based on SAA in table 4 and 5 is conservative than the IA and MC approaches. Both the sag and conductor length decrease with the increase of the environmental temperature, which drastically decreases the effect of the ice loading.

Table 6 and 7 show the DC and AC resistances in the span respectively. The resistance change for 20\% temperature uncertainty is found in table 1 . From table 1 input data, it is clear that when the temperature increases the resistance of the cable decreases.

The result in table 6 and 7 show not only the effect of temperature change on the change of the resistance value, but also the effect of OTL cable length change on the overall resistance of the cable in each span of the OTL. The SAA based total resistance value in the span based on the cable length in table 5 is slightly conservative than the other two methods. It has been discussed, as the SAA method is inclusive of both IA and MC based results since it considers uncertainties better than both of them.

Beside the computational advantage of IA over MC approach, in terms of memory space usage and convergence, the conservative nature of the end result is a point worth to be mentioned. A slightly more conservative result than the MC and IA approach is found with the application of SAA. In all the tables, the two probabilistic approach results are conserved inside the SAA results. The main reason behind its slight conservative of SAA over the other is, it keeps the relation between variables and preserves the correlation among them until the end result is found. Unlike IA, SAA does not suffer from dependency problem and take care of the round-off and truncation errors better than IA to provide worst case scenario results. Moreover, the $\mathrm{MC}$ approach considers the probability distribution function which underestimate the worst-case output.

Table 4. OTL Sag

\begin{tabular}{|c|c|c|c|}
\hline \multirow[t]{2}{*}{ Test Cases } & \multicolumn{3}{|l|}{ Sag (ft) } \\
\hline & $\mathrm{MC}$ & IA & AA \\
\hline 1 & {$[26.958,35.422]$} & {$[26.943,35.447]$} & {$[25.335,36.526]$} \\
\hline 2 & {$[25.601,33.489]$} & {$[25.570,33.515]$} & {$[24.037,34.552]$} \\
\hline 3 & {$[24.303,31.629]$} & {$[24.242,31.647]$} & {$[22.786,32.642]$} \\
\hline 4 & {$[22.993,29.833]$} & {$[22.961,29.844]$} & {$[21.583,30.794]$} \\
\hline 5 & {$[21.798,28.076]$} & {$[21.752,28.145]$} & {$[20.450,29.049]$} \\
\hline 6 & {$[20.583,26.433]$} & {$[20.569,26.477]$} & {$[19.347,27.334]$} \\
\hline 7 & {$[19.506,24.811]$} & {$[19.434,24.875]$} & {$[18.293,25.682]$} \\
\hline 8 & {$[18.368,23.283]$} & {$[18.346,23.338]$} & {$[17.289,24.093]$} \\
\hline 9 & {$[17.342,21.823]$} & {$[17.308,21.866]$} & {$[16.335,22.567]$} \\
\hline 10 & {$[16.359,20.395]$} & {$[16.319,20.460]$} & {$[15.432,21.105]$} \\
\hline 11 & {$[15.433,19.099]$} & {$[15.379,19.120]$} & {$[14.578,19.708]$} \\
\hline 12 & {$[14.522,17.813]$} & {$[14.489,17.846]$} & {$[13.774,18.376]$} \\
\hline 13 & {$[13.692,16.557]$} & {$[13.648,16.640]$} & {$[13.018,17.110]$} \\
\hline 14 & {$[12.890,15.454]$} & {$[12.856,15.500]$} & {$[12.310,15.911]$} \\
\hline 15 & {$[12.141,14.395]$} & {$[12.114,14.428]$} & {$[11.649,14.780]$} \\
\hline 16 & {$[11.466,13.387]$} & {$[11.419,13.422]$} & {$[11.031,13.718]$} \\
\hline 17 & {$[10.799,12.447]$} & {$[10.761,12.492]$} & {$[10.435,12.742]$} \\
\hline 18 & {$[10.165,11.603]$} & {$[10.140,11.636]$} & {$[9.8590,11.854]$} \\
\hline 19 & {$[9.5838,10.799]$} & {$[9.5617,10.843]$} & {$[9.3217,11.032]$} \\
\hline 20 & {$[9.0527,10.089]$} & {$[9.0256,10.111]$} & {$[8.8210,10.275]$} \\
\hline 21 & {$[8.5440,9.4086]$} & {$[8.5293,9.4373]$} & {$[8.3550,9.5792]$} \\
\hline 22 & {$[8.0914,8.8064]$} & {$[8.0709,8.8184]$} & {$[7.9220,8.9423]$} \\
\hline 23 & {$[7.6576,8.2287]$} & {$[7.6484,8.2513]$} & {$[7.5203,8.3610]$} \\
\hline 24 & {$[6.8474,7.1734]$} & {$[6.8421,7.1783]$} & {$[6.7524,7.2604]$} \\
\hline
\end{tabular}


Table 5. OTL Conductor Length

\begin{tabular}{|c|c|c|c|}
\hline \multirow[t]{2}{*}{ Test Cases } & \multicolumn{3}{|l|}{ Length (ft) } \\
\hline & MC & IA & AA \\
\hline 1 & {$[702.77,704.78]$} & {$[702.77,704.79]$} & {$[702.33,705.08]$} \\
\hline 2 & {$[702.50,704.27]$} & {$[702.49,704.28]$} & {$[702.10,704.55]$} \\
\hline 3 & {$[702.25,703.81]$} & {$[702.24,703.82]$} & {$[701.89,704.06]$} \\
\hline 4 & {$[702.01,703.39]$} & {$[702.01,703.39]$} & {$[701.69,703.61]$} \\
\hline 5 & {$[701.81,703.00]$} & {$[701.80,703.02]$} & {$[701.52,703.21]$} \\
\hline 6 & {$[701.61,702.66]$} & {$[701.61,702.67]$} & {$[701.37,702.85]$} \\
\hline 7 & {$[701.45,702.35]$} & {$[701.44,702.36]$} & {$[701.22,702.51]$} \\
\hline 8 & {$[701.29,702.07]$} & {$[701.28,702.07]$} & {$[701.09,702.21]$} \\
\hline 9 & {$[701.15,701.81]$} & {$[701.14,701.82]$} & {$[700.98,701.94]$} \\
\hline 10 & {$[701.02,701.58]$} & {$[701.01,701.59]$} & {$[700.88,701.70]$} \\
\hline 11 & {$[700.91,701.39]$} & {$[700.90,701.39]$} & {$[700.78,701.48]$} \\
\hline 12 & {$[700.80,701.21]$} & {$[700.80,701.21]$} & {$[700.70,701.29]$} \\
\hline 13 & {$[700.71,701.04]$} & {$[700.71,701.05]$} & {$[700.63,701.12]$} \\
\hline 14 & {$[700.63,700.91]$} & {$[700.63,700.92]$} & {$[700.56,700.96]$} \\
\hline 15 & {$[700.56,700.79]$} & {$[700.56,700.79]$} & {$[700.51,700.83]$} \\
\hline 16 & {$[700.50,700.68]$} & {$[700.50,700.69]$} & {$[700.46,700.72]$} \\
\hline 17 & {$[700.44,700.59]$} & {$[700.44,700.59]$} & {$[700.41,700.62]$} \\
\hline 18 & {$[700.39,700.51]$} & {$[700.39,700.52]$} & {$[700.37,700.54]$} \\
\hline 19 & {$[700.35,700.44]$} & {$[700.35,700.45]$} & {$[700.33,700.46]$} \\
\hline 20 & {$[700.31,700.39]$} & {$[700.31,700.39]$} & {$[700.29,700.40]$} \\
\hline 21 & {$[700.28,700.34]$} & {$[700.28,700.34]$} & {$[700.26,700.35]$} \\
\hline 22 & {$[700.25,700.30]$} & {$[700.25,700.30]$} & {$[700.24,700.30]$} \\
\hline 23 & {$[700.22,700.26]$} & {$[700.22,700.26]$} & {$[700.21,700.27]$} \\
\hline 24 & {$[700.18,700.20]$} & {$[700.18,700.20]$} & {$[700.17,700.20]$} \\
\hline
\end{tabular}

Table 6. OTL DC Resistance

\begin{tabular}{|c|c|c|c|}
\hline \multirow[t]{2}{*}{ Test Cases } & \multicolumn{3}{|l|}{$\operatorname{Rdc}(\mathrm{m} \Omega)$} \\
\hline & $\mathrm{MC}$ & IA & AA \\
\hline 1 & {$[18.481,18.749]$} & {$[18.477,18.753]$} & {$[18.465,18.760]$} \\
\hline 2 & {$[18.408,18.658]$} & {$[18.405,18.660]$} & {$[18.394,18.667]$} \\
\hline 3 & {$[18.335,18.565]$} & {$[18.334,18.569]$} & {$[18.325,18.575]$} \\
\hline 4 & {$[18.264,18.477]$} & {$[18.263,18.479]$} & {$[18.255,18.484]$} \\
\hline 5 & {$[18.196,18.385]$} & {$[18.194,18.390]$} & {$[18.186,18.395]$} \\
\hline 6 & {$[18.125,18.299]$} & {$[18.124,18.302]$} & {$[18.118,18.307]$} \\
\hline 7 & {$[18.058,18.214]$} & {$[18.055,18.215]$} & {$[18.050,18.219]$} \\
\hline 8 & {$[17.988,18.127]$} & {$[17.987,18.129]$} & {$[17.982,18.132]$} \\
\hline 9 & {$[17.920,18.042]$} & {$[17.919,18.044]$} & {$[17.915,18.047]$} \\
\hline 10 & {$[17.853,17.958]$} & {$[17.851,17.959]$} & {$[17.848,17.962]$} \\
\hline 11 & {$[17.785,17.874]$} & {$[17.784,17.875]$} & {$[17.781,17.877]$} \\
\hline 12 & {$[17.718,17.791]$} & {$[17.717,17.792]$} & {$[17.715,17.794]$} \\
\hline 13 & {$[17.651,17.708]$} & {$[17.651,17.709]$} & {$[17.649,17.711]$} \\
\hline 14 & {$[17.585,17.626]$} & {$[17.584,17.627]$} & {$[17.583,17.628]$} \\
\hline 15 & {$[17.519,17.545]$} & {$[17.518,17.545]$} & {$[17.517,17.546]$} \\
\hline 16 & {$[17.453,17.464]$} & {$[17.452,17.464]$} & {$[17.451,17.465]$} \\
\hline 17 & {$[17.380,17.390]$} & {$[17.379,17.390]$} & {$[17.379,17.391]$} \\
\hline 18 & {$[17.300,17.323]$} & {$[17.300,17.324]$} & {$[17.299,17.325]$} \\
\hline 19 & {$[17.220,17.258]$} & {$[17.220,17.258]$} & {$[17.219,17.259]$} \\
\hline 20 & {$[17.141,17.192]$} & {$[17.140,17.192]$} & {$[17.140,17.193]$} \\
\hline 21 & {$[17.061,17.126]$} & {$[17.061,17.127]$} & {$[17.061,17.127]$} \\
\hline 22 & {$[16.982,17.061]$} & {$[16.982,17.062]$} & {$[16.982,17.062]$} \\
\hline 23 & {$[16.903,16.996]$} & {$[16.903,16.996]$} & {$[16.902,16.997]$} \\
\hline 24 & {$[16.823,16.930]$} & {$[16.823,16.931]$} & {$[16.823,16.931]$} \\
\hline
\end{tabular}


Table 7. OTL AC Resistance

\begin{tabular}{lrrr}
\hline \multirow{2}{*}{ Test Cases } & Rac $(\mathrm{m} \Omega)$ & \multicolumn{1}{c}{} & \multicolumn{1}{c}{ AA } \\
\cline { 2 - 4 } & MC & IA & {$[27.051,27.431]$} \\
1 & {$[27.071,27.414]$} & {$[27.068,27.420]$} & {$[26.963,27.313]$} \\
3 & {$[26.980,27.301]$} & {$[26.978,27.303]$} & {$[26.876,27.197]$} \\
4 & {$[26.893,27.184]$} & {$[26.889,27.188]$} & {$[26.789,27.083]$} \\
5 & {$[26.804,27.069]$} & {$[26.801,27.075]$} & {$[26.704,26.971]$} \\
6 & {$[26.718,26.958]$} & {$[26.714,26.963]$} & {$[26.619,26.860]$} \\
7 & {$[26.630,26.851]$} & {$[26.628,26.853]$} & {$[26.534,26.750]$} \\
8 & {$[26.544,26.741]$} & {$[26.542,26.744]$} & {$[26.450,26.642]$} \\
9 & {$[26.460,26.635]$} & {$[26.457,26.637]$} & {$[26.367,26.535]$} \\
10 & {$[26.374,26.527]$} & {$[26.373,26.530]$} & {$[26.284,26.429]$} \\
11 & {$[26.290,26.424]$} & {$[26.289,26.425]$} & {$[26.201,26.324]$} \\
12 & {$[26.208,26.318]$} & {$[26.206,26.321]$} & {$[26.119,26.220]$} \\
13 & {$[26.125,26.216]$} & {$[26.123,26.217]$} & {$[26.037,26.117]$} \\
14 & {$[26.041,26.113]$} & {$[26.040,26.115]$} & {$[25.956,26.015]$} \\
15 & {$[25.959,26.012]$} & {$[25.958,26.013]$} & {$[25.875,25.913]$} \\
16 & {$[25.877,25.911]$} & {$[25.877,25.912]$} & {$[25.794,25.812]$} \\
17 & {$[25.796,25.810]$} & {$[25.795,25.811]$} & {$[25.704,25.721]$} \\
18 & {$[25.706,25.719]$} & {$[25.705,25.720]$} & {$[25.606,25.639]$} \\
19 & {$[25.608,25.637]$} & {$[25.607,25.638]$} & {$[25.508,25.557]$} \\
20 & {$[25.509,25.556]$} & {$[25.509,25.556]$} & {$[25.410,25.476]$} \\
21 & {$[25.411,25.475]$} & {$[25.411,25.475]$} & {$[25.313,25.395]$} \\
22 & {$[25.313,25.394]$} & {$[25.313,25.394]$} & {$[25.215,25.314]$} \\
23 & {$[25.216,25.313]$} & {$[25.216,25.314]$} & {$[25.020,25.152]$} \\
24 & {$[25.118,25.233]$} & {$[25.118,25.233]$} & \\
\hline
\end{tabular}

In order to show the self-validating nature of SAA, different percent of uncertainty is used and the result is depicted in Fig 2. A fixed output result of table 1 input without uncertainty is also simulated and plotted with uncertain results. Fig 2 result shows for any percent of uncertainty, the fixed input result is conserved around the mid of the uncertain input result. A higher percent of uncertainty results a wider bound and vice versa. For increasing temperature and pressure while ice thickness decreases the total weight decreases accordingly.
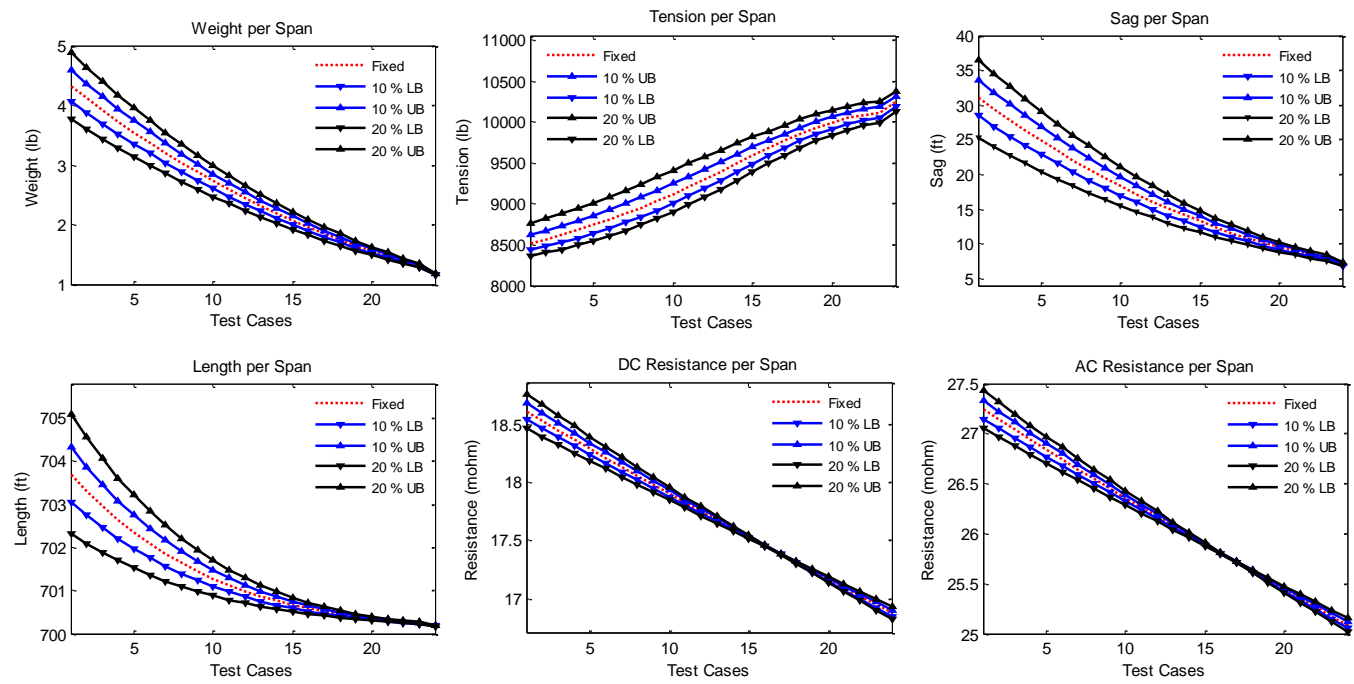

Fig.2. OTL Parameter Variation for Different Percent of Uncertainty 
In all the results of Fig 2, except the tension, the lower bounds result corresponds to lower bound input and the upper bound result corresponds to the upper bound input. For the particular case study input in table 1, when the value of temperature and wind pressure increases down from case one to twenty-four, the ice thickness decreases. The resultant weight in Fig 2 decreases from case one to twenty-four. This indicates that as the resultant weight depends highly on the ice thickness than the wind pressure for the particular case study. On the other hand, the OTL tension increases from case one to twenty-four and hence its dependency on the resultant weight is less than the temperature change. Since the sag is directly proportional to the decreasing resultant weight and inversely proportional to the increasing OTL tension, the final sag decreases from case one to twenty-four. Decreasing sag results decreasing conductor length. The DC and AC resistance from case one to twenty-four decreases according to the decreasing conductor length.

To elaborate the conservative nature of SAA over MC and IA approach, the maximum value of the deviation from the fixed result for different percent of uncertainty is shown in Fig 3. The result shows that the SAA based result is slightly conservative than the MC and IA based approaches for all percent of uncertainty considered. From the table values and graphs, it can be concluded that uncertainty based analysis gives flexibility of getting the worst-case results which includes all intermediate values whose inputs are bounded inside table 1 inputs for different diameter of uncertainty.
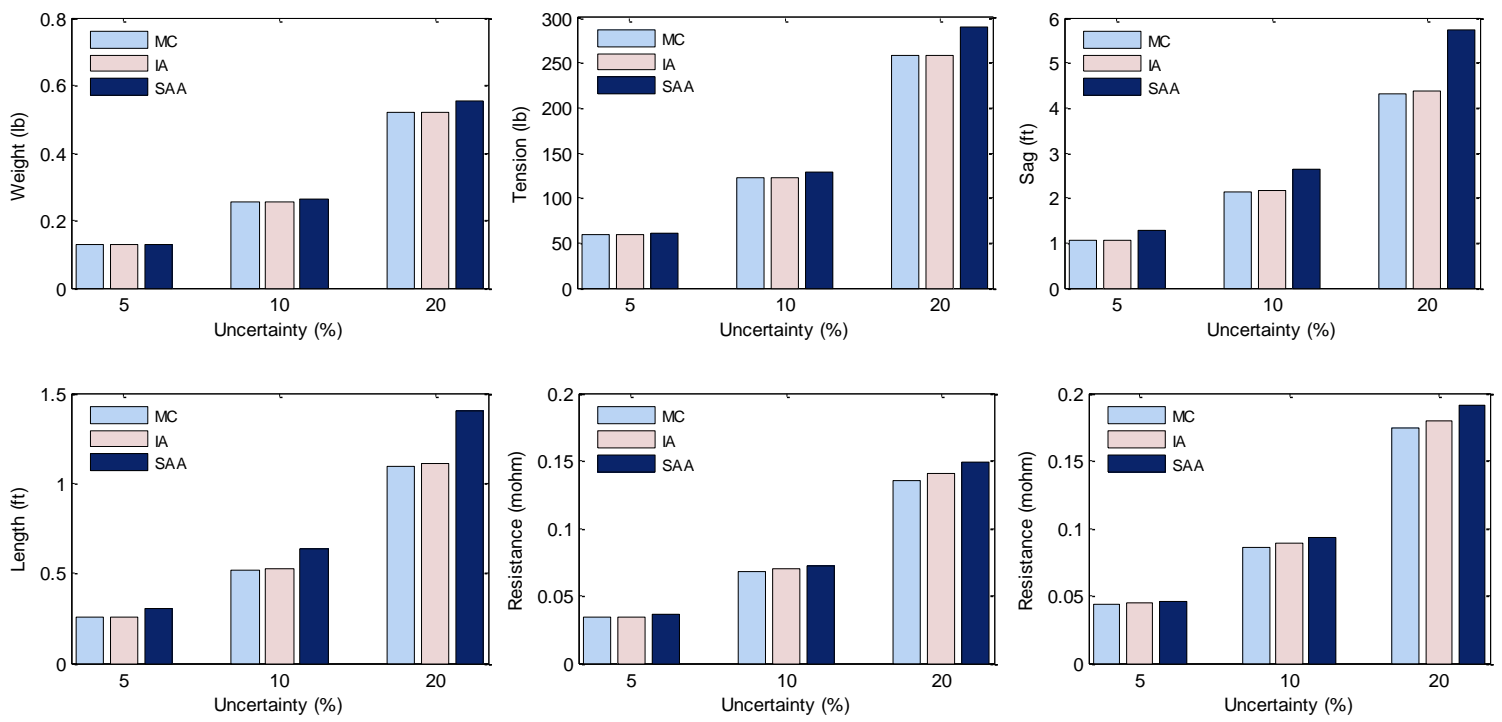

Fig.3. Maximum Uncertainty Deviation from the Fixed Result

\section{Conclusion}

Due to the advancement in smart grid technology, the old thinking about only load varies significantly than a generation and OTL parameters become an outdated topic.

The effect of temperature change on OTL overloading, sag, tension, cable length and resistances have been presented in this paper. The proposed SAA based approach is validated using MC and IA approach as a conservative algorithm than both of them. In terms of time consumption both SAA and IA simulation are effective than the probabilistic MC approach which takes 10,000 trials to converge. Based on the tabulated and graphed results, SAA approach is slightly conservative than IA and MC approaches. This is mainly due to its ability to consider all sorts of uncertainty and tracks the relationship between variable than IA and MC approaches. 
The variability of the results from case one to twenty-four shows that deterministic approach fails to give worst-case results of OTL parameters in a single calculation. The proposed algorithm can be extended to study the capacitance and inductance of OTL with temperature variation.

\section{References}

[1] L. Chen, Z. Zheng, S. Liu, L. Guo, and C. Dun, "Thermal grading around OTL under various environments and its influence to load capacity", 2012 IEEE International Symposium on Electrical Insulation, pp. 545-548, 2012.

[2] Yixun Shi, "A Probability Model for Occurrences of Large Forest Fires", International Journal of Engineering and Manufacturing (IJEM), vol. 1, no. 1, pp. 1-7, 2012

[3] C.-P. Meng and Y.-C. Cheng. "A New Ice Covering Model. 2008 International Conference on High Voltage Engineering and Application”, pp. 140-143, 2008.

[4] X. Meng, L. Hou, L. Wang, M. Macalpine, G. Fu, B. Sun, Z. Guan, W. Hu, and Y. Chen. "Oscillation of conductors following ice-shedding on UHV OTLs", Mechanical Systems and Signal Processing, vol. 30, pp. 393-406, 2012.

[5] J. Hou, Y. Li, and Z. Sun. "Unbalanced Tensions and Vertical Space Calculation of Tranmission Lines under Non-uniform Ice-coating and Ice-Shedding”, Energy Procedia, vol. 17, pp. 1034-1042, 2012.

[6] K. Tucker and A. Haldar. "Numerical Model Validation and Sensitivity Study of a Transmission-Line Insulator Failure Using Full-Scale Test Data", IEEE Transactions on Power Delivery, vol. 22, no. 4, pp. 2439-2444, 2007.

[7] R. M. Holmukhe, P. S. Chaudhari, P. P. Kulkarni, K. Deshpande, and P. Kulkarni. "Measurement of Weather Parameters via Transmission Line Monitoring System for Load Forecasting", 2010 3rd International Conference on Emerging Trends in Engineering and Technology, pp. 298-303, 2010.

[8] S. Malhara and V. Vittal. "Monitoring sag and tension of a tilted transmission line using geometric transformation”, 2009 IEEE Power \& Energy Society General Meeting, pp. 1-7, 2009.

[9] Manju Mam, Leena G, N.S. Saxena, "Distribution Network Reconfiguration for Power Loss Minimization Using Bacterial Foraging Optimization Algorithm", International Journal of Engineering and Manufacturing (IJEM), vol. 6, no.2, pp.18-32, 2016.

[10] J. Stolfi and L. D. Figueiredo. "An Introduction to Affine Arithmetic", TEMA - Tendências em Matemática Aplicada e Computacional, vol. 4, no. 3, pp. 297-312, 2003.

[11] L. H. D. Figueiredo and J. Stolfi, "Affine Arithmetic Concepts and Applications", Numerical Algorithms, vol. 37, no. 1-4, pp. 147-158, 2004.

[12] W. Heidrich, P. Slusallek, and H.-P. Seidel, "Sampling procedural shaders using affine arithmetic", ACM Transactions on Graphics, vol. 17, no. 3, pp. 158-176, Jan. 1998.

[13] F. Messine, "Extensions of Affine Arithmetic. Application to Unconstrained Global Optimization", Journal of Universal Computer Science, vol. 8, no. 11, pp. 992-1015, 2002.

[14] G. Spagnuolo and N. Femia. "True worst-case circuit tolerance analysis using genetic algorithms and affine arithmetic", IEEE Transactions on Circuits and Systems I: Fundamental Theory and Applications, vol. 47, no. 9, pp. 1285-1296, 2000.

[15] D.Das, Eletrical Power Systems, 1st Edition, New Delhi: New Age International publisher, 2006.

[16] L. L. Grigsby, Electric power generation, transmission, and distribution. Boca Raton: CRC Press, 2007.

[17] J. Slegers, "Transmission Line Loading: Sag Calculations and High-Temperature Conductor Technologies", Iowa State University, pp. 1-24, 2011.

[18] H. Shaalan, "Transmission line analysis using interval mathematics" 2012 North American Power Symposium (NAPS), pp.1-5, 2012.

[19] H. Shaalan, "Calculating transmission line inductance using interval mathematics" Probabilistic Methods Applied to Power Systems, 2004 International Conference on, pp. 224-226, 2004. 


\section{Authors' Profiles}

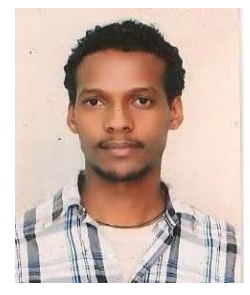

Yoseph Mekonnen Abebe received his MSc in Power System Engineering from Bahir Dar University, Ethiopia in 2013. He is currently a Ph.D candidate in Andhra University, Visakhapatnam, India. His area of research includes Overhead Transmission line and load flow analysis in the presence of input uncertainty.

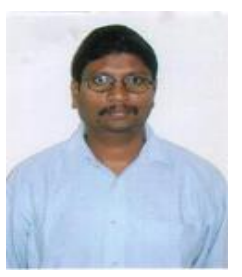

Mallikarjuna Rao Pasumarthi is a professor of Electrical Engineering in Andhra University College of engineering Visakhapatnam India. He has 16 years of teaching experience in the field of electrical engineering in general and control system engineering in particular. His area of research includes, design and analysis of specific purpose electrical machines and their controllers and uncertainty based power system analysis.

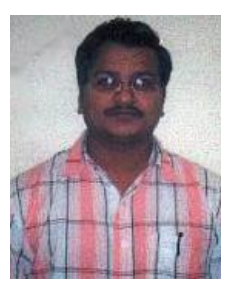

Gopichand Naik Mudavath, is an assistant professor of Electrical Engineering in Andhra University College of engineering Visakhapatnam India. His area of research includes, high voltage engineering and an uncertainty based power system network analysis.

How to cite this paper: Yoseph Mekonnen Abebea, P. Mallikarjuna Rao, M. Gopichand Naik,"Analysis of Overhead Transmission Line Parameters with Uncertainty", International Journal of Engineering and Manufacturing(IJEM), Vol.7, No.5, pp.16-30, 2017.DOI: 10.5815/ijem.2017.05.02 